\title{
La prueba preconstituida en el Instituto de Medicina Legal y Ciencias Forenses de Valencia: características de los delitos de abuso y agresión sexual
}

\section{The pre-constituted test in the Institute of Legal Medicine and Forensic Sciences of Valencia: characteristics of abuse and sexual assault crimes}

Fecha de recepción: 2-10-2017

Fecha de aceptación: 10-11-2017
Adriana Rey Anastasi

Psicóloga del Instituto de Medicina Legal y Ciencias Forenses. Valencia

Elena Martínez Miguel

Grado en Psicología en Prácticas en el Instituto de Medicina Legal y Ciencias Forenses. Valencia

Inmaculada Pedroche Garde

Psicóloga del Instituto de Medicina Legal y Ciencias Forenses. Valencia

\section{resumen/alostract:}

En el presente estudio hemos analizado el estado actual de la prueba preconstituida en el Instituto de Medicina Legal y Ciencias Forenses de Valencia, así como las características de los delitos de abuso y agresión sexual como delitos que con mayor frecuencia precisan de la realización de dicha prueba. Los resultados muestran una sobrerrepresentación femenina en las víctimas de abuso y agresión sexual. Los abusos sexuales se producen, generalmente, en víctimas que tienen entre 0 y 11 años, los agresores pertenecen al ámbito intrafamiliar, son continuados en el tiempo y se producen sin penetración. Las agresiones sexuales se producen, generalmente, en víctimas de entre 8 a 17 años, los agresores pertenecen al ámbito extrafamiliar, se repiten a lo largo del tiempo y se producen con penetración. Los resultados acerca de la prueba preconstituida reflejan el aumento progresivo en el número de peticiones e intervenciones. Además, se está produciendo la exportación de la prueba a otros delitos debido a la demanda, y hay un retraso temporal en su realización. Finalmente, de los 163 testimonios obtenidos mediante prueba preconstituida, 80 de ellas pueden ser consideradas como prueba de cargo a utilizar durante el juicio oral, pudiendo evitarse la asistencia de la víctima al mismo.

In the present study we have analyzed the current state of the pre-constituted test at the Institute of Legal Medicine and Forensic Sciences of Valencia, and the characteristics of the crimes of abuse and sexual assault as crimes that most frequently require the test. The results show a female over-representation in the victims of abuse and sexual assault. Sexual abuse usually occurs in victims between 0 and 11 years of age, the perpetrators belong to the family, are continued in time and occur without penetration. Sexual assaults usually occur in victims aged between 8 and 17 years, the perpetrators belong to the extrafamiliar sphere, are repeated over time and occur with penetration. For the pre-constituted test, the results reflect the progressive increase in the number of petitions and interventions. In addition, the export of the test to other crimes is occurring due to the demand, and there is a temporary delay in its realization. Finally, of the 163 pre-constituted tests carried oud, 80 of them can be considered as proof of charge to be used during the oral trial, and the victim's attendance at the trial can be avoided.

\section{palahras clave/keywords:}

prueba preconstituida; abuso sexual; agresión sexual; testimonio infantil

pre-constituted test; sexual abuse; sexual assault; child testimony 


\section{Introducción}

En España, el aumento de la concienciación social respecto a los delitos sufridos por víctimas especialmente vulnerables, ha dado lugar a la producción de importantes avances legislativos. Se considera víctima especialmente vulnerable aquella cuyo riesgo de victimización secundaria o victimización por el sistema judicial es mayor, y que además, debido a sus características, tiene disminuida su capacidad para defender sus propios derechos. Se trata de víctimas menores de edad o personas con la capacidad judicialmente modificada. La reforma introducida por la Ley Orgánica 8/2006, de 4 de diciembre, por la que se modifican los artículos 433, 448, 707 y 731 bis, regulando cómo tiene que llevarse a cabo la exposición de dichas víctimas al sistema judicial, y en consecuencia, aquellas medidas o aspectos que tienden a evitar la victimización secundaria, cuando tienen que declarar en juicio oral como víctimas de un delito. El artículo 433.3 dispone que el Juez de Instrucción, en el caso de los testigos menores de edad o personas con la capacidad judicialmente modificada, podrá acordar, cuando a la vista de la falta de madurez de la víctima resulte necesario para evitar causarle graves perjuicios, que se le tome declaración mediante la intervención de expertos y con intervención del Ministerio Fiscal. La Circular 3/2009 de la Fiscalía General del Estado, de 10 de noviembre, sobre protección de los menores víctimas y testigos, establece que será conveniente la realización de la prueba preconstituida en casos de menores víctimas de delitos sexuales cuando se acredite, mediante informe pericial, que la comparecencia a juicio oral provocará perjuicio psicológico en el menor; o cuando el menor sea muy pequeño, o el testimonio pueda verse deteriorado por el lapso de tiempo entre la primera declaración y la fecha del juicio oral (Sotoca, Muñoz, González y Manzanero, 2013). Es un referente jurisprudencial la Sentencia $n^{\circ}$ 96/2009 del Tribunal Supremo (Sala 2a), pues introduce la posibilidad de impedir la comparecencia en el juicio oral de menores de edad víctimas de delitos sexuales, otorgando validez como prueba de cargo preconstituida a las declaraciones prestadas por el menor durante la fase de instrucción. Aunque no se dispone de un estatuto de protección de los menores en el proceso penal cuando actúan en calidad de víctimas o testigos (González, Muñoz, Sotoca y Manzanero, 2013), la prueba preconstituida se ha ido asentando como un recurso jurídico posible, con dos objetivos fundamentales: proteger el testimonio de la víctima del posible deterioro provocado por múltiples e inadecuados abordajes; y evitar la victimización secundaria provocada por el sistema policial y judicial (González et al., 2013; Sotoca et al., 2013; Alcón y Montalvo, 2011).

\section{La prueba preconstituida.}

Los delitos contra víctimas especialmente vulnerables son problemáticos, sobretodo cuando son difíciles de demostrar, como ocurre con los abusos sexuales (González et al., 2013), puesto que la mayoría se producen sin testigos y sin evidencias físicas que los acrediten (Manzanero, 1996; Muñoz, González-Guerrero, Sotoca, Terol, González y Manzanero, 2016; Echeburúa y Subijama, 2008). Cuando el juez carece de indicios físicos u objetivos debe conceder importancia a indicios más subjetivos para acreditar los hechos denunciados (Scott, Manzanero, Muñoz y Köhnken, 2014). El testimonio de la víctima (huella de memoria o indicio cognitivo), que será el principal medio de prueba con el que contará el 
juez (Vázquez-Rojas, 2014), es extremadamente sensible al paso del tiempo y a la metodología utilizada para la obtención del testimonio, sobre todo en aquellos casos en los que las víctimas son menores de más corta edad (Muñoz et al., 2016). Según la Circular 3/2009 de la Fiscalía General del Estado sobre protección de menores víctimas y testigos, la edad límite para el testimonio infantil se situa alrededor de los tres años. Resulta extremadamente importante que se guarde una cadena de custodia similar a la que se sigue con los indicios físicos, para evitar que los recuerdos puedan alterarse, sustituirse por otros, contaminarse o destruirse (González et al., 2013). La entrevista forense se ha convertido en la principal técnica de obtención del indicio cognitivo en menores víctimas de abuso sexual (Diges y Pérez, 2017).

La realización temprana de la prueba preconstituida evita la pérdida de nitidez del recuerdo por el paso del tiempo, además de evitar posibles alteraciones producidas por el entorno en el que convive el menor. La grabación de la declaración permite una posterior reproducción del recuerdo sin que éste se vea afectado por numerosas entrevistas y permite valorar si el estilo de entrevista del entrevistador ha sido el adecuado. Diversas investigaciones han demostrado que la forma en que la toma de declaración es conducida por el profesional afectará a la cantidad de información relatada por el menor y a la validez de su declaración (García, 2013; Diges y Pérez, 2017). Por ello, resultan de especial relevancia las aportaciones realizadas desde la Psicología del Testimonio, que han potenciado el desarrollo de diversas técnicas de entrevista (González et al., 2013), científicamente contrastadas, que permiten obtener un relato espontáneo, amplio y preciso de los delitos que están siendo investigados. Los protocolos de actuación utilizados durante la conducción de la prueba preconstituida deben tener en cuenta que el objetivo de la entrevista cognitiva adaptada al ámbito forense es obtener la máxima cantidad de información relativa a los hechos denunciados. Para ello, el entrevistador debe tener la mente abierta a hipótesis alternativas (sin focalizar la entrevista a confirmar los abusos sexuales) y debe evitar la realización de preguntas sugerentes o coactivas puesto que pueden contaminar el testimonio de la víctima (Diges y Pérez, 2017).

En julio de 2014, el Decanato de los Juzgados de Valencia pone en marcha la práctica de la prueba preconstituida. Desde entonces, la Unidad de Psicología Forense del Instituto de Medicina Legal y Ciencias Forenses de la Comunidad de Valencia (conformada por dos psicólogas forenses), asume, cuando así lo requiere el juez, la conducción de dicha prueba en una sala equipada con un sistema audiovisual. Este sistema evita la confrontación directa con el acusado y la frialdad y formalidad de la sala y el juicio en sí. La metodología empleada por la Unidad de Psicología Forense de Valencia coincide, en gran medida, con un modelo de entrevista cognitiva mejorada, el Modelo PEACE (Milne, Shaw y Bull, 2007). En 1993, dicho modelo, que fue creado por el psicólogo Eric Sheperd, supuso un cambio radical en la manera de llevar a cabo las investigaciones. Fue creado originariamente para ser utilizado por la policía de Inglaterra y Gales, sin embargo, ha sido adoptado por diferentes países (García, 2013). Bajo el mismo esquema, el Modelo PEACE (mnemotecnia para las distintas fases que lo componen: Planning and preparation; Engage and explain; Account; Closure; Evaluation) puede ser aplicado a imputados, víctimas y testigos (Da Graça-Ballardin, 2010). Las diversas fases deben ser conceptualizadas como una "caja de herramientas", 
pues le corresponde al entrevistador seleccionar aquellas técnicas que mejor se adecuen a la investigación en cada caso (Paulo, Albuquerque y Bull, 2014).

El testimonio prestado por la víctima durante la realización de la prueba preconstituida tendrá valor probatorio y podrá utilizarse durante el juicio oral cuando se determine que el menor es competente para prestar declaración, y que dicha declaración es válida.

La falta de confianza respecto a la competencia del menor para prestar declaración se sustenta en falsas creencias (Unicef, 2015), que determinan que a los niños les cuesta diferenciar la fantasía de la realidad, que mienten, que no son capaces de recordar detalladamente cómo acontecieron los hechos o que son fácilmente influenciables. Es difícil que un niño que no tiene conocimientos sobre una actividad sexual, pueda fantasear detalles sobre ella. Resulta imprescindible que el técnico que entreviste al menor evalúe su capacidad para prestar declaración, y para ello debe determinar: si el menor que ha sido supuestamente víctima de un delito de abuso sexual es capaz de comprender lo que dice, de expresarlo correctamente y con sus propias palabras; si posee las competencias psíquicas que determinan su capacidad para declarar; si está dispuesto a ofrecer un testimonio que se ajuste a los sucesos y descartar la sugestionabilidad o facilidad para ser influenciado por terceros (Rey, 2016). El testimonio de la víctima será válido cuando la información contenida en él esté relacionada con el hecho y los sujetos que se está investigando, y esté oriendado a la búsqueda de la verdad material (Barrios, 2005). Las declaraciones válidas, que están basadas en registros de memoria, deben diferenciarse de las que son producto de la invención, la fantasía o la influencia de otra persona (Godoy-Cervera y Higueras, 2005).

\section{El abuso sexual infantil.}

No existe una definición única de abuso sexual infantil (ASI), sin embargo, existe cierto consenso sobre su conceptualización. Le definición aportada por el Acta para la Prevención y Tratamiento del Maltrato Infantil (U.S. Department of Health and Human Services, 2010), es bastante amplia, pues lo definen como a. la utilización, la persuasión, la inducción, la seducción o la coerción de un niño o niña para realizar [o participar de] - incluida la ayuda a otra persona para el mismo fin - cualquier tipo de conducta sexual explícita, o la simulación de dicha conducta con el fin de producir una representación visual de esta; o b. la violación, el tocamiento, la prostitución o cualquier otra forma de explotación sexual de un niño o niña, o el incesto. Con la puesta en vigor de la reforma del Código Penal operada por la Ley Orgánica 1/2015, de 30 de marzo, los Delitos contra la libertad e indemnidad sexual, recogidos en el Título VIII del Libro II, ayudan a determinar, dentro de la concepción de abuso sexual, las siguientes categorías (Save the Children, 2001): abuso sexual, agresión sexual, acoso sexual, exhibicionismo, prostitución, explotación sexual y corrupción de menores. Se puede considerar abuso o agresión sexual cualquier forma de contacto físico, con o sin acceso carnal (pudiendo haberse producido el acceso carnal por vía vaginal, anal o bucal, o mediante la introducción de miembros corporales u objeto por alguna de las dos primeras vías). La diferencia básica estriba en que para que concurra la agresión sexual, el que atenta contra la libertad sexual de la víctima lo ha hecho utilizando violencia o intimidación, mientras que en el caso del abuso sexual, no hay violencia ni intimidación, pero sî engaño o falta de consentimiento. 
La investigación del abuso sexual infantil se ve limitada por aquellos casos que no llegan al sistema judicial, registrándose, en consecuencia, los casos de mayor gravedad. Aunque no puede calcularse con precisión el número de casos de abuso sexual infantil, los estudios coinciden en afirmar que los abusos sexuales perpetrados contra víctimas menores de edad son más frecuentes de lo que la sociedad concibe (Save The Children, 2001; Unicef, 2015). Esta elevada cifra negra de criminalidad (Lameiras y Orts, 2014) se debe principalmente a que el abuso sexual infantil se produce en la máxima intimidad, conociéndose únicamente los casos que se denuncian. El meta-análisis realizado por Stoltenborgh, Van Ijzendoorn, Euser y Bakermans-Kranenburg (2011) determina que las estadísticas a escala mundial reflejan una "sobrerrepresentación femenina" dentro de las víctimas de abuso sexual infantil. En cuanto a la edad de las víctimas, las investigaciones han determinado que hay dos intervalos de edad que suponen un mayor riesgo para el abuso sexual (Unicef, 2015; Echeburúa y Guerricaechevarría, 2005): entre los 6 y 7 años y entre los 10 y los 12 (coincidiendo este último intervalo con el inicio del desarrollo de las características sexuales). En España, el informe publicado por el Centro Reina Sofía (2011) y el publicado por el Centro Reina Sofía en colaboración con la Valencian International University (2011) sobre la situación del menor víctima en la Comunitat Valenciana, determinan que la mayoría de casos se encuentran entre los 8 y 11 años, que suele ser continuado en el tiempo (tiende a repetirse una vez comienza), y que el agresor suele ser un familiar o conocido de la víctima. El mayor número de abusos sexuales son cometidos por padres, padrastros y parejas sentimentales de la madre (Unicef, 2015), pues tienen un acceso más fácil al menor, más oportunidades de poder continuar los abusos, y mayor dependencia del menor respecto al abusador teniendo en cuenta la relación familiar.

El objetivo del presente estudio es doble: por un lado, analizar la situación del recurso jurídico de la prueba preconstituida en la Unidad de Psicología Forense del Instituto de Medicina Legal y Ciencias Forenses de Valencia, y por otro, analizar las características principales de los delitos de abuso y agresión sexual, pues son los delitos contra la libertad e indemnidad sexual en los que más se ven implicados los menores de edad como víctimas, y los que con mayor frecuencia precisan de la realización de la prueba preconstituida.

\section{Método}

\section{Muestra.}

La lectura de los expedientes judiciales que han entrado en la Unidad de Psicología Forense del Instituto de Medicina Legal y Ciencias Forenses de Valencia como petición de prueba preconstituida ha permitido obtener información relativa a 243 sujetos. Del total, 157 eran mujeres y 86 hombres, con edades comprendidas entre los 2 y los 17 años en el momento de realización de la denuncia.

\section{Instrumentos.}

Los datos se recopilaron analizando toda la información obrante en el expediente judicial: denuncia, declaración de la víctima, del acusado y de los testigos del delito; partes médicos del servicio de urgencias; historiales clínicos de las unidades de salud mental; informes pe- 
riciales psicológicos realizados a las víctimas, generalmente por asociaciones como CAVAS o Espill; e informes periciales realizados por médicos forenses. Se analizaron las grabaciones de las pruebas preconstituidas realizadas, y los informes periciales emitidos tras la realización de la prueba con el objetivo de valorar la credibilidad del testimonio prestado por la víctima.

\section{Procedimiento.}

El análisis de los expedientes, de las grabaciones y de los informes, permitió obtener información acerca de las siguientes variables relativas a las pruebas preconstituidas:

- Tipo de delito por el que se ha solicitado la petición.

- Razón por la que no se han realizado determinadas pruebas preconstituidas.

- Sexo de la víctima.

- Edad de la víctima en el momento de interposición de la denuncia.

- Edad de la víctima en el momento de realización de la prueba preconstituida.

- Tiempo que pasa desde que se realiza la petición a la Unidad de Psicología Forense hasta que se lleva a cabo.

- Método de obtención del testimonio.

- Método de valoración del testimonio.

- Competencia de la víctima para prestar declaración.

- Validez de la declaración.

- Consideración del testimonio como prueba de cargo.

Además, para valorar las características de los delitos de abuso y agresión sexual se ha recogido información acerca de las siguientes variables:

- Tipo de delito (abuso o agresión sexual).

- Edad de la víctima en el inicio de los abusos.

- Sexo de la víctima.

- Denunciante.

- Relación existente entre víctima y agresor (abuso extrafamiliar o intrafamiliar).

- Producido con o sin acceso carnal.

- Frecuencia de los hechos denunciados.

- Duración de los hechos denunciados.

\section{Análisis de datos.}

Los datos se han analizado mediante el paquete estadísticos SPSS 22.0. Se han calculado frecuencias, porcentajes y medias; y se ha realizado comparación de medias empleando el análisis estadístico t de Student. 


\section{Resultados}

Desde julio de 2014 hasta julio de 2017 han llegado a la Unidad de Psicología Forense del Instituto de Medicina Legal y Ciencias Forenses de Valencia 243 peticiones de pruebas preconstituidas, de las que han sido realizadas 163. En la Tabla 1 puede observarse que el $22.5 \%$ de las pruebas, correspondientes al año 2017, estaban pendientes de ser realizadas en el momento de la recogida de datos.

Las tres razones principales por las que no se han llevado a cabo las pruebas son: que se trata de víctimas de delitos no referidos a delitos contra la libertad e indemnidad sexual (20\%), que el juzgado deja sin efecto la realización de la misma (13.8\%) y que el menor está victimizado y su testimonio está contaminado (12.5\%).

Puede observarse en la Figura 1 que 2016 ha sido el año en el que más peticiones de prueba preconstituida han llegado a la Unidad de Psicología Forense y en el que más pruebas se han llevado a cabo. Hasta julio de 2017, de las 37 peticiones que habían entrado, estaban aceptadas y pendientes de ser realizadas 18 pruebas preconstituidas.

Una vez la petición llega a la Unidad de Psicología Forense, pasan una media de 110.86 días $(\mathrm{DT}=73.25)$ hasta que la prueba preconstituida es realizada (aprox. 4 meses). De las pruebas que se han llevado a cabo (véase Figura 2), el mayor porcentaje se han realizado a víctimas de delitos de abuso sexual (58.9\%), seguido de las realizadas a víctimas de delitos de malos tratos en el ámbito familiar (11\%) y las realizadas a víctimas de delitos de malos tratos en el ámbito escolar (9.2\%). También se han realizado pruebas preconstituidas a menores de edad que no eran víctimas directas del delito, sino que prestaban su declaración como supuestos testigos del delito.

En el momento de realización de la prueba preconstituida, la media de edad de los sujetos es de 9.36 (DT = 3.38). De los 163 sujetos, 103 eran mujeres y 60 hombres, con edades comprendidas entre los 3 y los 17 años. La mayoría de pruebas preconstituidas se han realizado a sujetos con edades comprendidas entre los 8 y los 17 años (65.6\%).

Competencia para prestar declaración, validez de los testimonios y consideración del testimonio como prueba de cargo.

Los técnicos que entrevistaron a los sujetos durante la realización de la prueba preconstituida determinaron que del total de sujetos entrevistados, el $74.8 \%$ de ellos eran competentes para prestar declaración. La media de edad de los sujetos competentes es de 9.95 (DT = 3.18), ligeramente superior a la media de edad de los sujetos no competentes $(\mathrm{M}=7.61$; DT $=3.39$ ). En este sentido, y en lo que concierne a las diferencias de edad en el momento de realización de la prueba según la competencia para prestar declaración, estas eran significativas: $\mathrm{t}_{(163)}=3.88 ; \mathrm{p}=0.00$. Del total de testimonios obtenidos en las pruebas realizadas, el $54.6 \%$ se han valorado como válidos. Los resultados reflejan que el $54.6 \%$ de los testimonios obtenidos mediante prueba preconstituida tienen valor probatorio, y podrían ser utilizados como prueba durante el juicio oral.

El abuso y la agresión sexual como delitos contra la indemnidad sexual que con más frecuencia precisan de la realización de la prueba preconstituida. 


\section{Características del abuso sexual.}

De los 129 casos de abuso sexual registrados, el 93.8.\% eran delitos de abuso sexual y el $6.2 \%$ delitos de abuso sexual unidos a delitos de malos tratos en el ámbito familiar. Los datos reflejan una sobre-representación femenina en las víctimas de abuso sexual: el $72.1 \%$ de las víctimas eran mujeres y el $27.9 \%$ hombres. En cuanto a la edad, en el inicio de los abusos sexuales, la media de edad es de 8.04 ( $\mathrm{DT}=3.15)$. En el caso de las mujeres, la media es de 8.33 ( $\mathrm{DT}=3.02)$, superior a la de los hombres $(\mathrm{M}=7.21$; $\mathrm{DT}=3.39)$. Puede observarse en la Tabla 2, que la edad de los menores víctimas de abuso sexual se concentra en el intervalo de edad de 8 a 11 años (45.3\%) seguido del intervalo de edad de 0 a 7 años (40.6\%).

En la mayoría de los casos registrados, la madre de la víctima es quien interpone la denuncia (68.2\%). Según la relación entre la víctima y el agresor, gran parte de los abusos sexuales son intrafamiliares $(61.2 \%)$. Dentro del ámbito familiar, el mayor porcentaje de agresores son los padres de las víctimas (31.6\%), seguido de las parejas sentimentales de las madres con convivencia (20.2\%), y de los abuelos (13.9\%). Fuera del ámbito familiar el mayor porcentaje de agresores son desconocidos (28\%) y amigos de la familia (22\%).

En cuanto a la duración, es más representativo que los abusos sean continuos y que perduren desde el inicio de los mismos (44.2\%), que esporádicos (34.9\%). La duración media de los abusos sexuales continuos (evaluada en meses de duración) es de $16.28(\mathrm{DT}=17.09)$ y la duración media de los abusos sexuales esporádicos (evaluada en episodios concretos) es de 1.27 (DT = 0.72). Por último, en cuanto a la distribución de los abusos sexuales teniendo en cuenta que se hayan producido con o sin acceso carnal, la mayoría de las víctimas afirma que los abusos se han producido sin penetración (72.9\%). Además, si tenemos en cuenta el sexo de la víctima de abuso sexual, de los 34 casos producidos con penetración, 22 eran mujeres (el 71\%) y 9 hombres (el 29\%).

\section{Características de la agresión sexual.}

De los 20 casos de agresión sexual registrados, el 92.3\% eran delitos de agresión sexual, y sólo el 7.7\% delitos de agresión sexual unidos a delitos de malos tratos en el ámbito familiar. La mayoría de las agresiones sexuales se han producido en mujeres (95\%). En cuanto a la edad, en el inicio de las agresiones, la media de edad es de $10.65(\mathrm{DT}=3.05)$. En las mujeres, la edad media es de $10.79(\mathrm{DT}=3.06)$, superior a la edad de 8 años que tenía el único hombre víctima de agresión sexual registrado. El mayor porcentaje de víctimas se encuentra en el intervalo de edad de 8 a 11 años (45\%), seguido del intervalo de 12 a 17 años (35\%). Puede observarse en la Tabla 3 que en la mayoría de los casos registrados es la madre quien interpone la denuncia (80\%). Existe un mayor porcentaje de abusos extrafamiliares (55\%) que intrafamiliares (45\%). Dentro del ámbito familiar, el mayor porcentaje de agresores son las parejas sentimentales de las madres con convivencia (44.4\%) seguidos de los padres (22.2\%). Fuera del ámbito familiar, el mayor porcentaje de agresores son los amigos de la familia (33.3\%) y los desconocidos $(27.3 \%)$.

El mayor porcentaje de agresiones sexuales suelen ser continuadas en el tiempo (65\%). La duración media de las agresiones sexuales continuas (evaluada en meses de duración) es de $16(\mathrm{DT}=15.9)$ y la de las agresiones sexuales esporádicas (evaluadas en episodios concre- 
tos) es de $1.43(\mathrm{DT}=0.78)$. Por último, la mayoría de las víctimas afirma que las agresiones sexuales se han producido con penetración (85\%). Si tenemos en cuenta el sexo de la víctima de abuso sexual, de los 17 casos producidos con penetración, 16 eran mujeres (el 94.1\%) y sólo uno de ellos era hombre (el 5.9\%).

\section{DISCUSIÓN}

El aumento en el número de solicitudes para la práctica de la prueba preconstituida y en el número de pruebas realizadas por la Unidad de Psicología Forense del Instituto de Medicina Legal y Ciencias Forenses de Valencia desde julio de 2014 reflejan el proceso de consolidación de la prueba preconstituida como recurso jurídico posible. Un recurso, que según la Circular 3/2009 es recomendable utilizar en menores de edad víctimas de delitos sexuales, puesto que debido a sus características (producidos en la intimidad, sin testigos ni evidencias físicas que los corroboren) el testimonio de la víctima se convierte en el principal medio de prueba con el que contará el juez. Esta situación ha llevado a que se admita legalmente el recurso a la prueba preconstituida en este tipo de delitos.

La adecuada realización de la prueba exige conocimientos del funcionamiento de la memoria y formación especializada en técnicas cognitivas de entrevista de obtención y valoración del testimonio. La utilización de protocolos específicos de entrevista forense facilita la obtención de testimonios libres de sesgos, tanto cognitivos como procedimentales (Muñoz et al., 2016). Los técnicos de la Unidad de Psicología Forense han optado por el Modelo PEACE de Milne, Shaw y Bull (2007), un protocolo científicamente aceptado de entrevista forense que permite incrementar la cantidad de información correcta sin que aumente el número de información distorsionada o contaminada.

El correcto uso de la técnica permite determinar la competencia del testigo para prestar declaración y la validez de la misma; pues sólo cuando se dan ambas condiciones se podrá considerar el testimonio como prueba de cargo o descargo, por la autoridad judicial. Del estudio realizado se desprende que, de los 163 testimonios obtenidos mediante prueba preconstituida por la Unidad de Psicología Forense, 80 de ellos podrían considerarse poseen valor probatorio; siento este un resultado muy alentador para la continuidad de esta práctica jurídica.

En cuanto a la competencia del testigo, los resultados del presente estudio reflejan que la mayoría de menores eran competentes para prestar declaración, y que la edad de los sujetos competentes era significativamente superior a la de los no competentes. Estos resultados pueden deberse a que la edad es un factor condicionante de la capacidad testifical de los menroes víctimas de abuso sexual (Rey, 2016). Sin embargo, no podemos olvidar que existen otros factores que limitan la competencia del menor como testigo: el tiempo (la duración de los procedimientos judiciales afecta a la calidad y a la exactitud del recuerdo, y dificulta la recuperación emocional de la víctima), la sugestionabilidad, la fabulación o incapacidad para distinguir sucesos vividos de los inventados, la inducción externa y consciente por parte de un adulto que lleve a las víctimas a denunciar y mantener alegaciones falsas, el aprendizaje post-evento (la adquisición de conocimientos sexuales impropios para la edad de la víctima tras sucesivas entrevistas), el estrés e incluso la adecuación de la entrevista. 
La virtualidad o bondades de esta práctica procesal no está, por lo demás, exenta de dificultades u obstáculos, siendo evidente que no todos los resultados obtenidos son favorables, debido entre otros motivos, a la escasez de recursos personales y materiales. Entre los problemas advertidos destaca el excesivo tiempo que transcurre entre la petición de la prueba y la realización de la misma (una media aproximada de 4 meses).

Este dato preocupante se debe, según consideramos, a diferentes motivos: el elevado número de peticiones; la escasa disponibilidad de tiempo (teniendo en cuenta que las dos psicólogas de la Unidad combinan las funciones periciales propias de su cargo con la práctica de las pruebas preconstituidas); fallos tecnológicos (sonido, imagen, grabación, que determinan la repetición y/o suspensión de la prueba); desconocimiento del funcionamiento de la prueba desde el ámbito judicial (ausencia de algunas de las partes del procedimientos: letrados, investigado); y por último, en ocasiones, el estado emocional de la víctima impide la realización de la prueba en el día señalado, con el consiguiente aplazamiento.

La mayoría de estudios revisados se centran en la descripción de las características principales del abuso sexual infantil como una conceptualización que engloba el abuso sexual, la agresión sexual, el acoso sexual, el exhibicionismo, la provocación sexual, la prostitución, la explotación sexual infantil y la corrupción de menores. Estudiar las características de las víctimas y de los agresores de diferentes delitos como si fueran idénticos y fueran a arrojar los mismos resultados impide que se vean las diferencias entre ellos, dificultando la prevención, detección e intervención en los diferentes delitos contra la libertad e indemnidad sexual. Es por ello, que en este estudio se han analizado las características de los delitos de abuso y agresión sexual de manera independiente.

Los delitos de abuso y agresión sexual constituyen los dos principales delitos contra la libertad e indemnidad sexual por los que se solicitan y se realizan más pruebas preconstituidas. Los resultados del presente estudio reflejan similitudes y diferencias entre los mismos. Los casos registrados de abuso sexual reflejan una sobrerrepresentación femenina dentro de las víctimas. En cuanto a la edad, los resultados han determinado que hay dos intervalos de edad donde se concentran la mayoría de casos: de 8 a 11 años, seguido del intervalo de 0 a 7 años. La persona que interpone la denuncia suele ser la madre de la víctima. Los abusos sexuales son, en su mayoría intrafamiliares, perpetuados por los padres de las víctimas seguido de las parejas sentimentales de la madre con convivencia. El abuso sexual intrafamiliar provoca un conflicto de lealtades (Unicef, 2015). No sólo están pendientes de un hilo las relaciones afectivas entre el agresor y la víctima, sino también las del agresor con el resto de miembros de la familia. La relación de dependencia respecto a los padres y las parejas de la madre con convivencia provocan que la víctimas, que conocen las consecuencias de desbelar los sucedido, se mantengan en silencio. El vínculo emocional existente con la víctima, facilita la utilización del engaño y la manipulación como formas de iniciar y mantener los abusos. No hay forma de que la revelación del abuso sexual intrafamiliar no produzca un inevitable desorden emocional (Magaña, Ramírez, y Menéndez, 2013). El mayor porcentaje de abusos registrados son continuados en el tiempo, con una media de duración superior a los 16 meses. Los abusos sexuales suelen producirse sin penetración, y es más habitual que se produzca el acceso carnal cuando las víctimas son mujeres. 
Los casos registrados de agresión sexual también reflejan una sobrerrepresentanción femenina dentro de las víctimas, pero superior a las víctimas de abuso sexual infantil, pues sólo hay un caso registrado de agresión sexual donde la víctima fuera un hombre. Los resultados también han determinado que hay dos intervalos de edad donde se concentran la mayoría de los casos: de 8 a 11 años, seguido del intervalo de 12 a 17 años. La media de edad de las víctimas, situada alrededor de los 11 años, coincide con el inicio del desarrollo de las características sexuales. La madre suele ser quien interpone la denuncia. Es más frecuente que las agresiones sexuales sean extrafamiliares, producidas en la mayoría de los casos por amigos de la familia seguido de personas desconocidas. Cuando el perpetrador es conocido por la familia, esta relación funciona como una vía de acceso a la víctima, y al mantenimiento del secreto. La menor vinculación emocional respecto a la víctima facilita la utilización, por parte de los desconocidos, de formas de manipulación agresivas, prevalenciendo así la violencia y la intimidación como formas de tener acceso a la víctima. El mayor porcentaje de casos registrados son continuados en el tiempo, con una media de duración superior a 16 meses, y con acceso carnal.

Para terminar, han de realizarse algunas reflexiones respecto a las limitaciones de este estudio. Aunque el objetivo principal era determinar el cumplimiento de los objetivos de la prueba preconstituida (preservar el testimonio y evitar la victimización secundaria), éste tuvo que modificarse, debido, principalmente, a la imposibilidad de contactar con cada uno de los juzgados responsables en cada momento del procedimiento (Juzgado de Instrucción, Juzgado de lo Penal o Audiencia Provincial). Además, el hecho de que algunos casos no estuvieran resueltos en el momento de la recogida de datos, impediría conocer dicho resultado. Sería interesante que los técnicos encargados de llevar a cabo las pruebas preconstituidas y los operadores jurídicos encargados de solicitarlas aunaran esfuerzos para evaluar el verdadero cumplimiento de los objetivos de la prueba preconstituida y el uso adecuado de la misma.

\section{REFERENCIAS}

Alcón, M. F., y Montalvo, F. (2011). Los menores en el proceso judicial: La protección del menor frente al derecho a un juicio justo. Madrid: Tecnos.

Barrios, B. (2005). El testimonio penal. Panamá: Editorial Jurídica Ancón.

Centro Reina Sofía. (2011). Maltrato infantil en la familia en España. Madrid.

Centro Reina Sofía, Valencian International University. (2011). Informe sobre la situación del menor (víctima e infractor) en la Comunitat Valenciana. Valencia.

Da Graça-Ballardin, M. (2010). A entrevista investigativa e o policial entrevistador. Tesis doctoral, Pontifícia Universidade Católica Do Rio Grande Do Sul, Porto Alegre.

Diges, M., y Pérez, N. (2017). La entrevista forense de investigación a niños supuestas víctimas de delitos sexuales: guía de buenas prácticas. Diario La Ley (8919).

Echeburúa, E., y Guerricaechevarría, C. (2005). Abuso sexual en la infancia: Víctimas y agresores. Barcelona: Ariel.

Echeburúa, E., y Subijama, I. J. (2008). Guía de buena práctica psicológica en el tratamiento judicial de los niños abusados sexualmente. International Journal of Clinical and Health Psychology , 8 (3), 733-749. 
García, F. (2013). Análisis comparativo de protocolos de entrevista investigativa con niños, niñas y adolescentes que han sido víctimas de delitos sexuales. Tesis doctoral, Universidad de Chile, Departamento de Psicología, Santiago de Chile.

Garrido, E., y Masip, J. (2004). La evaluación del abuso sexual infantil. Congreso de Psicología Jurídica y Forense en Red. Salamanca: Universidad de Salamanca.

Godoy-Cervera, V., y Higueras, L. (2005). El Análisis de Contenido Basado en Criterios (CBCA) en la evaluación de la credibilidad del testimonio. Papeles del Psicólogo, 26, 92-98.

González, J. L., Muñoz, J. M., Sotoca, A., y Manzanero, A. L. (2013). Propuesta de protocolo para la conducción de la prueba preconstituida en víctimas especialmente vulnerables. Papeles del Psicólogo , 34 (3), 227-237.

Lameiras, M., y Orts, E. (2014). Delitos sexuales contra menores, abordaje psicológico, jurídico y policial. Valencia: Tirant Lo Blanc.

Manzanero, A. L. (1996). Evaluando el testimonio de menores testigos y víctimas de abuso sexual. Anuario de Psicología Jurídica, 6, 13-34.

Milne, R., Shaw, G., y Bull, R. (2007). Investigative interviewing: The role of psychology. En D. Carson, R. Milne, F. Pakes, y K. Shalev, Applying psychology to criminal justice (págs. 65-80). Chichester, UK: BPS Blackwell.

Muñoz, J. M., González-Guerrero, L., Sotoca, A., Terol, O., González, J. L., y Manzanero, A. L. (2016). La entrevista forense: Obtención del indicio cognitivo en menores presuntas víctimas de abuso sexual infantil. Papeles del Psicólogo , 37 (3), 205-2016.

Paulo, R. M., Albuquerque, P. B., y Bull, R. (2014). A Entrevista Cognitiva Melhorada: Pressupostos teóricos, investigação e aplicação. Revista da Associação Prtuguesa de Psicologia , 28 (2), 21-30.

Rey, A. (2016). Peritaje penal en menores: El menor víctima de abuso sexual. Universidad Católica de Valencia.

Save the Children. (2001). Abuso sexual infantil: Manual de formación para profesionales.

Scott, M. T., Manzanero, A. L., Muñoz, J. M., y Köhnken, G. (2014). Admisibilidad en contextos forenses de indicadores clínicos para la detección del abuso sexual infantil. Anuario de Psicología Jurídica , 24, 57-63.

Sotoca, A., Muñoz, J. M., González, J. L., y Manzanero, A. M. (2013). La prueba preconstituida en casos de abuso sexual infantil: Aportaciones desde la psicología jurídica. La Ley Penal, 102, 112-122.

Steller, M., y Köhnken, G. (1989). Statement analysys: Credibility assessment of children's testimonies in sexual abuse cases. En D. C. Raskin, Psychological methods in criminal investigation and evidence (págs. 217-245). Nueva York: Springer.

Stoltenborgh, M., Van ljzendoorn, M. H., Euser, E. M., y Bakermans-Kranenburg, M. J. (2011). A global perspective on child sexual abuse: meta-analysis of prevalence around the world. Child Maltreatment , 16 (2), 79-101.

U.S. Department of Health and Human Services. (2010). The Child Abuse Prevention and Treatment Act, Including Adoption Opportunities \& The Abandoned Infant Asistante Act

Unicef. (2015). Abuso Sexual Infantil: Cuestiones relevantes para su tratamiento en la justicia. Montevideo.

Vázquez-Rojas, C. (2014). Sobre la cientificidad de la prueba científica en el proceso judicial. Anuario de Psicología Jurídica , 24 (1), 65-73. 
Tabla 1. Distribución de los motivos de no realización de la prueba preconstituida

\begin{tabular}{lcc}
\hline & $\mathrm{n}$ & $\%$ \\
\hline Pendiente de realización en el momento de recopilación & 18 & 22.5 \\
Delito no referido a delitos contra la libertad e indemnidad sexual & 16 & 20 \\
Juzgado deja sin efecto la petición de la prueba preconstituida & 11 & 13.8 \\
Víctima victimizada y testimonio contaminado & 10 & 12.5 \\
Juzgado deja sin efecto la petición de la prueba preconstituida y pide en su & 7 & 8.8 \\
lugar un informe pericial & & 7.5 \\
Víctima demasiado pequeña para declarar & 6 & 6.3 \\
Es supuesto testigo del delito, no víctima del mismo & 5 & 3.8 \\
La víctima no acude a la citación & 3 & 1.3 \\
Se suspende porque la víctima se niega a declarar & 1 & 1.3 \\
Las partes implicadas no quieren continuar con la prueba & 1 & 1.3 \\
Víctima no localizable & 1 & 1.3 \\
Sobreseimiento & 80 & 100 \\
TOTAL PETICIONES NO REALIZADAS & & \\
\hline
\end{tabular}

Figura 1. Número de peticiones de pruebas preconstituidas y número de pruebas preconstituidas realizadas desde julio de 2014 hasta julio de 2017.

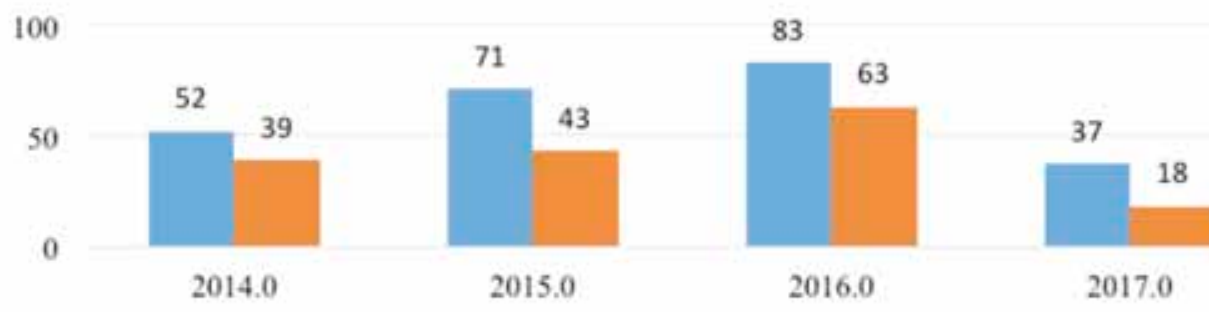

m $N$ o peticiones $\quad$ № $\mathrm{p}$. preconstituidas realizadas 
Figura 2. Porcentaje de pruebas preconstituidas que han sido realizadas por cada tipo de delito.

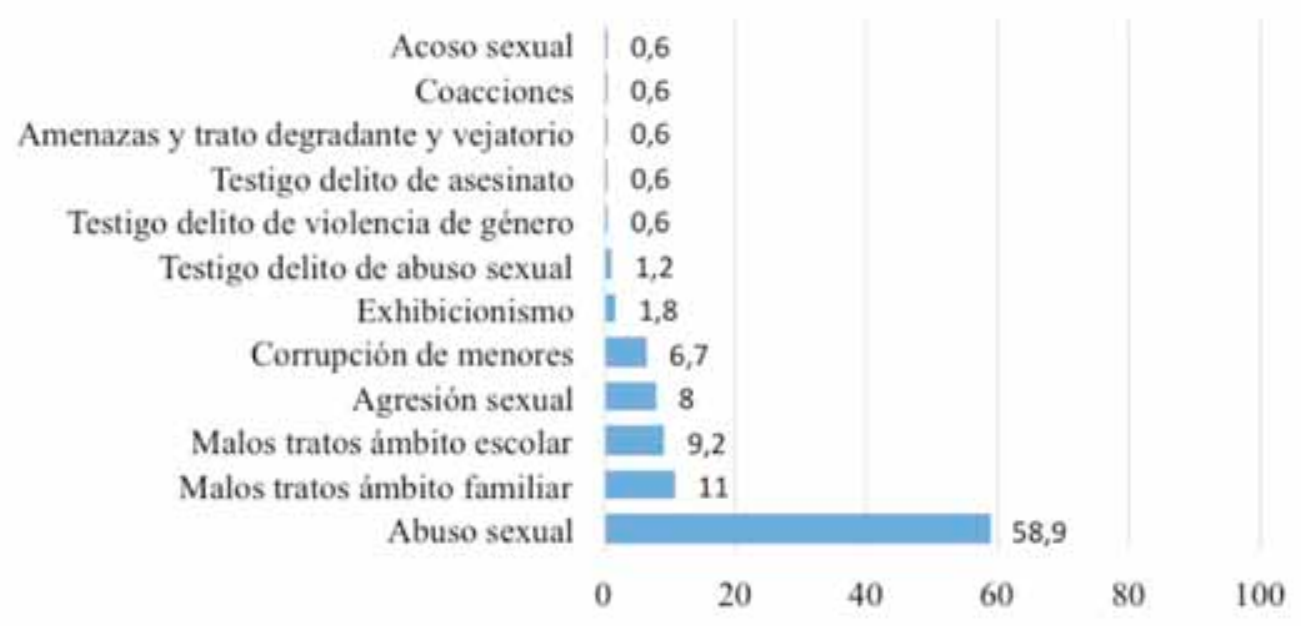


Tabla 2. Distribución de las características de los delitos de abuso sexual

\begin{tabular}{|c|c|c|}
\hline & $\mathrm{n}$ & $\%$ \\
\hline \multicolumn{3}{|l|}{ Edad de las víctimas } \\
\hline 0-7 años & 43 & 40.6 \\
\hline 8-11 años & 48 & 45.3 \\
\hline 12-17 años & 15 & 14.1 \\
\hline TOTAL & $106^{*}$ & 100 \\
\hline \multicolumn{3}{|l|}{ Denunciante } \\
\hline Progenitores & 2 & 1.6 \\
\hline Madre & 88 & 68.2 \\
\hline Padre & 10 & 7.8 \\
\hline Víctima & 8 & 6.2 \\
\hline Policía Nacional & 1 & 0.8 \\
\hline Fiscalía de Menores & 7 & 5.4 \\
\hline Prima & 1 & 0.8 \\
\hline Director Centro Docente & 5 & 3.9 \\
\hline Abuela & 1 & 0.8 \\
\hline Amiga de la familia & 3 & 2.3 \\
\hline Servicios Sociales & 1 & 0.8 \\
\hline Psicóloga & 1 & 0.8 \\
\hline Directora Centro de Acogida & 1 & 0.8 \\
\hline TOTAL & 132 & 100 \\
\hline \multicolumn{3}{|c|}{ Tipo de abuso según la relación agresor-víctima } \\
\hline Extrafamiliar & 50 & 38.8 \\
\hline Intrafamiliar & 79 & 61.2 \\
\hline TOTAL & 129 & 100 \\
\hline \multicolumn{3}{|l|}{ Duración } \\
\hline Continuo & 57 & 44.2 \\
\hline Esporádico & 45 & 34.9 \\
\hline Desconocido $* *$ & 24 & 18.6 \\
\hline La víctima niega los abusos & 3 & 2.3 \\
\hline TOTAL & 129 & 100 \\
\hline $\begin{array}{l}* 23 \text { datos desconocidos } \\
* * \text { No aparece información relativ }\end{array}$ & & \\
\hline
\end{tabular}


Tabla 3. Distribución de las características de los delitos de agresión sexual

\begin{tabular}{lcc}
\hline & $\mathrm{n}$ & $\%$ \\
\hline Edad de las víctimas & 4 & 20 \\
$0-7$ años & 9 & 45 \\
$8-11$ años & 7 & 35 \\
$12-17$ años & 20 & 100 \\
\hline TOTAL & 16 & 80 \\
\hline Denunciante & 4 & 20 \\
$\quad$ Madre & 20 & 100 \\
$\quad$ Víctima & & \\
TOTAL & 11 & 55 \\
\hline Tipo de abuso según la relación agresor-víctima & 9 & 45 \\
$\quad$ Extrafamiliar & 20 & 100 \\
$\quad$ Intrafamiliar & & 65 \\
TOTAL & 13 & 35 \\
\hline Duración & 7 & 100 \\
$\quad$ Continuo & 20 & \\
Esporádico & 20 & \\
TOTAL &
\end{tabular}

